\title{
Hepatic pulmonary fusion: a rare case report
}

\author{
Yewei Xie \\ Department of Pediatric Cardiothoracic Surgery, Shanghai Children's Hospital, Shanghai Jiao Tong University, Shanghai, China \\ Correspondence to: Yewei Xie. Department of Pediatric Cardiothoracic Surgery, Shanghai Children's Hospital, Shanghai Jiao Tong University, \\ Shanghai 200062, China. Email: xieywsd88288@163.com.
}

\begin{abstract}
Hepatic pulmonary fusion (HPF) is a very rare congenital disease which is characterized by a fibrous connection between the liver and lung tissues. It is commonly associated with congenital diaphragmatic hernia (CDH), pulmonary sequestration, congenital heart disease and other diseases. Surgical operation has been reported to be the only option for the treatment of this disease. The most sophisticated point lies in how to define the dividing line between liver and lung fusion tissues. And the postoperative mortality is high. At present, the etiology and pathogenesis of HPF are not completely clear. In this study, we reported a case of a 4-month-old male infant presented with cough and shortness of breath and intraoperatively found to have HPF associated with atrial septal defect and scimitar syndrome. Staged surgery was performed to avoid the simultaneous involvement of multiple organs such as heart, lung and liver, and shorten the operation and anesthesia time to a certain extent, improving the success rate of the operation. We only separated the fusion tissues and repaired the diaphragmatic hernia in the first operation, and in the second surgery, we conducted intra-cardiac repair of cardiac malformations. The follow-up results showed that the right lung gradually developed and there were no significant abnormalities in liver. This experience can provide a useful reference for future cases.
\end{abstract}

Keywords: Hepatic pulmonary fusion (HPF); congenital heart malformation; congenital diaphragmatic hernia $(\mathrm{CDH})$; staged surgery; case report

Submitted Oct 29, 2020. Accepted for publication Feb 18, 2021.

doi: $10.21037 / \mathrm{tp}-20-356$

View this article at: http://dx.doi.org/10.21037/tp-20-356

\section{Introduction}

Hepatiac pulmonary fusion (HPF) is a very rare congenital malformation. The connection of the fiber bundles between the liver and lung or partial fusion of the liver and lung are both belong to HPF which is usually associated with other malformations including congenital diaphragmatic hernia $(\mathrm{CDH})$, congenital heart disease, ectopic drainage of pulmonary vein and isolated lung. Children with HPF are often accompanied with several respiratory symptoms such as cough and shortness of breath (1). The most sophisticated point lies in how to separate the liver and lung tissues completely. Here, we presented a 4-month-old male infant diagnosed with HPF (Figure 1) undergoing staged surgery. We present the following case in accordance with the CARE reporting checklist (available at http://dx.doi. org/10.21037/tp-20-356).

\section{Case presentation}

A 4-month-old male infant whose weight was $3 \mathrm{~kg}$ was admitted to our hospital because of coughing for more than one week, with poor general conditions including drowsiness, poor response, shortness of breath, poor skin elasticity, and poor growth. This male term infant was the first pregnancy for a nonconsanguineous couple. Although his thorax was symmetrical, weak breathing sound was observed in the upper right chest and thick breathing sound was observed in the left lung. I-II/VI SM (systolic murmur) was audible in the precardiac area, $2 \mathrm{~cm}$ below the liver costa. As shown in the chest computed tomography (CT) image (Figure 1A), part of the liver tissue herniated into the right chest cavity with right diaphragmatic hernia, and the diaphragm was not clearly defined, indicating the HPF of this infant. Cardiac ultrasonography suggested that 

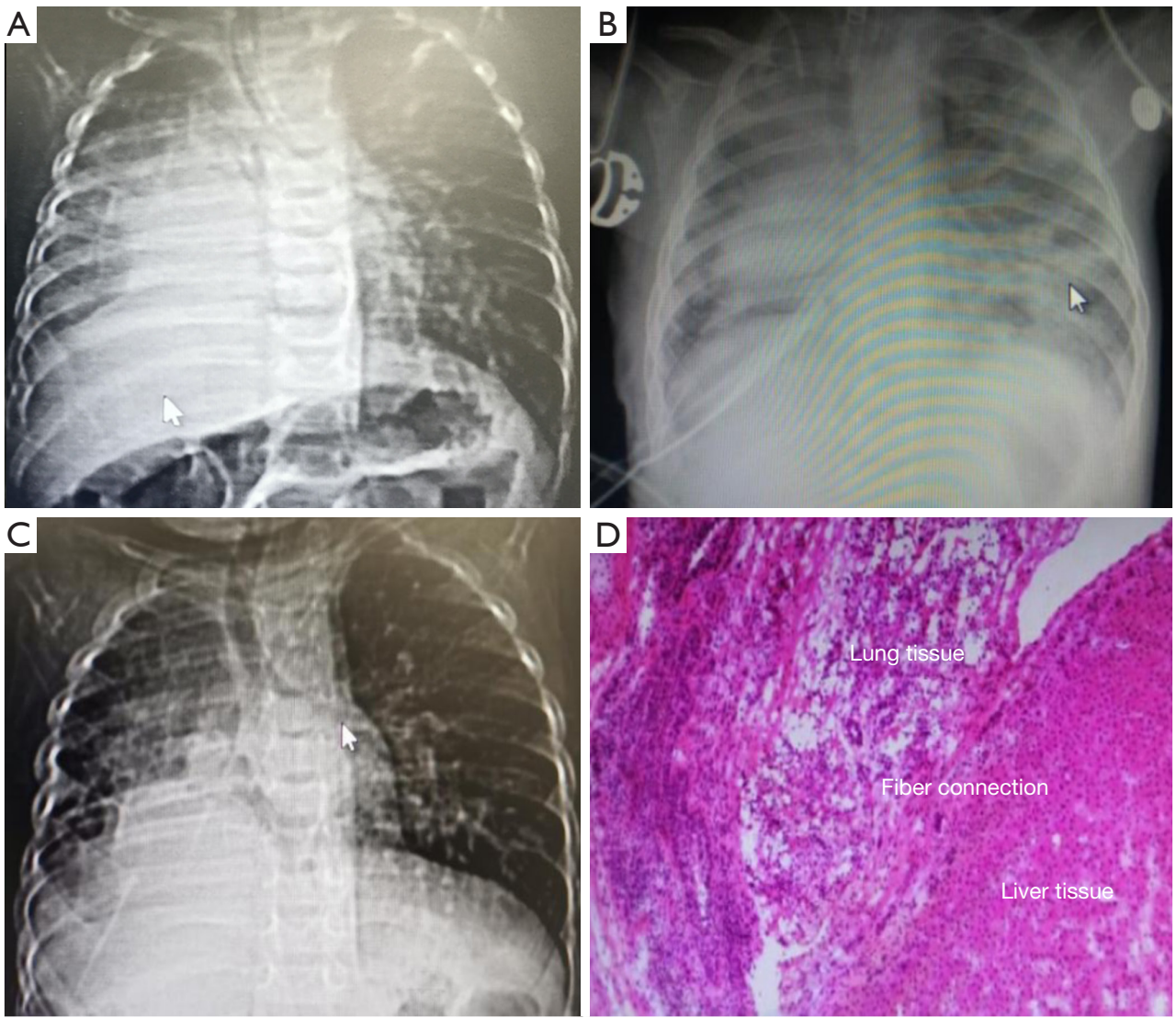

Figure 1 Chest radiograph, CT and histological examination of this patient: (A) chest CT image before the first operation, (B) chest radiograph on the day of the first operation, (C) chest CT image before the second operation, (D) histological examination with hematoxylin-eosin (HE) staining (original magnification: 4x) in the first operation. CT, computed tomography.

the infant had atrial septal defect and scimitar syndrome. Antispasmodic, antiasthmatic, phlegm reducing, and antiinflammatory treatments were given to this infant after admission. And multiple factors were taken into account for the choice of surgical options. On the one hand, the infant was too young to endure the long-time one-stage radical surgery. On the other hand, there was a paradox. We routinely used coagulants to avoid a massive hemorrhage in the division of HPF. But anticoagulants such as heparin were essential for patients accepted open heart surgery under cardiopulmonary bypass (CPB) for the prophylaxis of clots. Therefore, staged surgery was delivered to this infant at last. The first operation was conducted to separate the fusion tissues and repair the diaphragmatic hernia. And in the second surgery, we repaired the cardiac malformations.

The first operation was conducted on the $11^{\text {th }}$ day after admission through one incision on the right-side chest skin. The operation took about 6 hours and the amount of hemorrhage was $200 \mathrm{~mL}$. The elevated right lateral part of the diaphragm was observed during the operation. The abnormal hyperplasia of liver tissue entered the chest through the defective diaphragm and grew with the right lower lung. No distinct diaphragm was found in the abnormal liver and lung tissues. The right lung in the right chest was small, and the right upper lobe was healthy. The liver and lung fusion tissues were separated by the electrotome through the $\mathrm{V}$ intercostal space to return the abnormally proliferated liver tissue into the abdominal cavity. Artificial polyester patch was used to repair the defect of the diaphragm and reconstruct the right thoracic cavity through the VIII intercostal space. Postoperative bedside chest radiograph showed that the right upper lobe was larger than that before surgery, and the position of the right diaphragm was clearer (Figure $1 B$ ). As shown in Figure $1 D$, dysplastic alveoli companied with bronchial structure, bronchial mucosa and cartilage tissues can be 

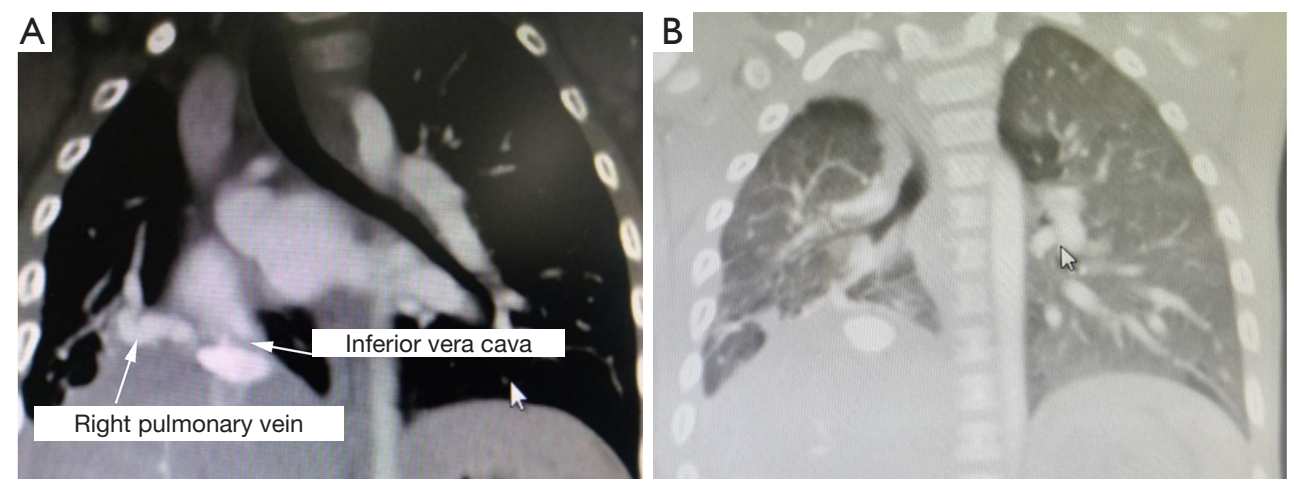

Figure 2 CTA and follow-up CT of this patient: (A) CTA before the second operation, (B) chest CT image 1 year after the first operation. CTA, computed tomography angiography; CT, computed tomography.

found adjacent to the liver tissue with patchy necrosis, supporting the diagnosis of HPF in this patient. The patient was discharged on day $22^{\text {th }}$ post operation.

The second surgery was performed when the infant was 17-month-old. His thorax was asymmetrical, and his right chest developed poorly with slightly weak breathing sounds before the second surgery. I-II/VI SM was audible in the precardiac area, $1 \mathrm{~cm}$ below the liver costa. Chest CT before the second operation showed that the right lung tissue grew significantly compared with that before the first operation (Figure 1C). He still had atrial septal defect and scimitar syndrome according to the cardiac ultrasonography. The computed tomography angiography (CTA) (Figure 2A) showed the right pulmonary vein entered directly into the inferior vena cava. The second operation was conducted through the $\mathrm{V}$ intercostal space on the right side into the chest. The smaller right pulmonary artery was observed during the operation. The right pulmonary veins formed the combined pulmonary vein, which descended from the right side, entering the inferior vena cava and the right atrium. The secondary atrial septal defect was $10 \mathrm{~mm}$ in diameter and located above the openings of the inferior vena cava and the right pulmonary vein. We reconstructed the tunnel with its own pericardial patch and drained the right pulmonary vein blood flow to the left atrium, at the same time, repairing the atrial septal defect. The operation took about 5 hours and the amount of hemorrhage was $100 \mathrm{~mL}$.

According to the postoperative test, the right lung gradually developed (Figure $2 B$ ), and there were no significant abnormalities in liver development and function. No adverse events occurred during the follow-up.

All procedures performed in studies involving human participants were in accordance with the ethical standards of the institutional and/or national research committee(s) and with the Helsinki Declaration (as revised in 2013). Written informed consent was obtained from the patient's parents.

\section{Discussion}

HPF is a very rare congenital malformation with a very low incidence. The children who suffer from HPF are always accompanied by respiratory symptoms such as repeated cough and shortness of breath. Katz et al. reported the first case of HPF in 1998 (2). There were only 14 literatures about HPF by the year of 2012 (3). According to the literatures, surgery is the only option for the treatment of this congenital disease. The difficulty of the surgery is that the dividing line between liver and lung fusion tissues cannot be clearly defined. In our opinion, we should divide these two kind tissues by both sight and touch in the operation. All of the supply of blood oxygen, the return of blood, the recovery of the normal blood flow in those two divided tissues should be taken into account. At the same time, the diaphragm muscles in the chest and abdominal cavity should be repaired. In the operation of this patient, we found the fusion of his liver and lung tissues. The intraoperative and postoperative pathological reports were described under the microscope. The histological examination in our case supported the diagnosis of HPF in this patient. There are some literatures which have reported the complete repair of the diaphragm defect. Artificial patches or autologous diaphragms were used to repair the defect of the diaphragm without segmenting liver and lung fusion tissues (4). In our case, the diaphragm was repaired with polyester patch. It has been reported that 
the postoperative mortality of HPF patients is high (5), due to hemorrhage, respiratory failure and heart failure. In our case, the infant had obvious respiratory symptoms before the operation. Staged operation was delivered to this infant. In the first operation, only the fusion tissues were separated to recovery the functions of the divided liver and lung tissues. And the ventilator was successfully evacuated on the third day after the first operation. We conducted the second surgery one year later, in which the corrective procedure for congenital heart defects (atrial septal defect, Scimitar syndrome) were performed. With the increase of the age and weight and the development of the right lung, the resistance of this infant improved, generating a better operation result (6). As shown in the postoperative bedside chest radiograph and chest CT, the right lung tissue grew significantly compared with that before operation indicating the gradual development of the remaining right lung.

At present, the definite etiology and pathogenesis of HPF are not clear. HPF is usually accompanied by other malformations such as $\mathrm{CDH}$, circulation abnormalities, isolated lungs, congenital heart disease, etc. In this case, the child with HPF had both right diaphragmatic hernia and congenital heart deformity (atrial septal defect, Scimitar syndrome). HPF without right diaphragmatic hernia has not been reported (3). Most of CDH occur in the left chest, and only $15 \%$ of diaphragmatic hernias occur in the right diaphragm. Therefore, some researchers suggested that if an infant had these characteristics including repeated cough, shortness of breath, a large opaque shadow above the right chest diaphragm in radiograph, no gastrointestinal shadow and dysplastic right lung, the infant was highly suspected of suffering from HPF (7). Other researchers speculated that the hypoplasia of the diaphragm leaded to the abnormal growth of the liver tissue, resulting in its entrance into the chest cavity and fusion of lung tissues (8). Several studies have revealed that the formation of diaphragmatic hernia was the result of congenital folding of the peritoneal muscular, which was not related to the development of the right lung (9). The relationship between the right lung dysplasia and the malformation of the embryonic layer was observed in animal experiments (10). Besides, genetic mutations were found in HPF, which affected the development of the mesoderm, leading to the formation of hepatopulmonary fusion and diaphragmatic hernia. Many genes were reported to play important roles in hepatopulmonary fusion, among which HGF (hepatocyte growth factor) was the most important (11).

In this case, we conducted staged surgery. In the first operation, we separated the fusion tissue and repaired the diaphragmatic hernia. And in the second surgery, we performed intra-cardiac repair of cardiac malformations for further treatment. The staged treatment strategy can not only avoid the simultaneous surgical involvements of multiple organs such as heart, lung and liver but also shorten the operation and anesthesia time to a certain extent, ensuring the success of the operation, which can provide a reference for future cases. And this child's parents appreciated the doctors very much for the appropriate treatments of their child.

\section{Acknowledgments}

Funding: Shanghai Medical Guidance and Technology Support Project (17411969000).

\section{Footnote}

Reporting Checklist: The author has completed the CARE reporting checklist. Available at http://dx.doi.org/10.21037/ tp-20-356

Conflicts of Interest: The author has completed the ICMJE uniform disclosure form (available at http://dx.doi. org/10.21037/tp-20-356). The author has no conflicts of interest to declare.

Ethical Statement: The author is accountable for all aspects of the work in ensuring that questions related to the accuracy or integrity of any part of the work are appropriately investigated and resolved. All procedures performed in studies involving human participants were in accordance with the ethical standards of the institutional and/or national research committee(s) and with the Helsinki Declaration (as revised in 2013). Written informed consent was obtained from the patient's parents.

Open Access Statement: This is an Open Access article distributed in accordance with the Creative Commons Attribution-NonCommercial-NoDerivs 4.0 International License (CC BY-NC-ND 4.0), which permits the noncommercial replication and distribution of the article with the strict proviso that no changes or edits are made and the original work is properly cited (including links to both the formal publication through the relevant DOI and the license). See: https://creativecommons.org/licenses/by-nc$\mathrm{nd} / 4.0 \%$. 


\section{References}

1. Chandrashekhara SH, Bhalla A, Gupta AK, et al. Hepatic pulmonary fusion: case report with review of literature. $\mathrm{J}$ Pediatr Surg 2011;46:e23-7.

2. Katz S, Kidron D, Litmanovitz I, et al. Fibrous fusion between the liver and the lung: an unusual complication of right congenital diaphragmatic hernia. J Pediatr Surg 1998;33:766-7.

3. Lin J, Durham MM, Ricketts R, et al. Hepatic pulmonary fusion: two cases with diaphragmatic hernia and one case with Pentalogy of Cantrell. Fetal Pediatr Pathol 2012;31:401-9.

4. Laamiri R, Belhassen S, Ksia A, et al. Right Congenital Diaphragmatic Hernia Associated With Hepatic Pulmonary Fusion: A Case Report. J Neonatal Surg 2016;5:35.

5. Botden SM, Heiwegen K, van Rooij IA, et al. Bilateral congenital diaphragmatic hernia: prognostic evaluation of a large international cohort. J Pediatr Surg 2017;52:1475-9.

Cite this article as: Xie Y. Hepatic pulmonary fusion: a rare case report. Transl Pediatr 2021;10(4):1034-1038. doi: $10.21037 /$ tp-20-356
6. Clugston RD, Greer JJ. Diaphragm development and congenital diaphragmatic hernia. Semin Pediatr Surg 2007;16:94-100.

7. Gander JW, Kadenhe-Chiweshe A, Fisher JC, et al. Hepatic pulmonary fusion in an infant with a rightsided congenital diaphragmatic hernia and contralateral mediastinal shift. J Pediatr Surg 2010;45:265-8.

8. Taide DV, Bendre PS, Kirtane JM, et al. Hepatic pulmonary fusion: a rare case. Afr J Paediatr Surg 2010;7:28-9.

9. Meeker TM. Pentalogy of Cantrell: reviewing the syndrome with a case report and nursing implications. J Perinat Neonatal Nurs 2009;23:186-94.

10. Nicolini U, Cerri V, Groli C, et al. A new approach to prenatal treatment of extralobar pulmonary sequestration. Prenat Diagn 2000;20:758-60.

11. Murata $S$, Nakata $M$, Sumie $M$, et al. Prenatal diagnosis of pentalogy of cantrell with craniorachischisis by threedimensional ultrasonography in the first trimester. Taiwan J Obstet Gynecol 2009;48:317-8. 